\title{
Nutrition matters
}

\author{
Fiona Godlee editor in chief, The BMJ
}

Nutrition has not traditionally been embraced by doctors as core to their practice. Our training and culture have led us to view food and dietary advice as the province of quacks, cranks, and faddists. Now, however, doctors are faced every day with the fallout of the global obesity epidemic and the chronic diseases that go with it.

In this, as in many complex issues in medicine, doctors have a triple role: to support the individual patient; where appropriate, to set an example through their own behaviour; and to understand and act on the underlying causes. Only if we do all three can we hope to make a difference.

Supporting and treating patients who are overweight or obese can be a challenge, as the updated guidance from the UK National Institute for Health and Clinical Excellence (NICE) acknowledges (doi:10.1136/bmj.g6608). The guidance carries few surprises. Diet and exercise remain the mainstay of lifestyle change. But the advice is now more cautious about the use of very low calorie diets and clearer about referrals for bariatric surgery. Patients with a BMI above 35 and recent onset type 2 diabetes should be referred, it says, but only if they will be assessed in a specialist weight management service within a tertiary referral centre and followed up in the long term.

As for setting an example by our own behaviour, this can be more easily said than done. It makes sense that doctors and other health professionals should keep fit and manage their own weight properly, for their own sake and to give greater credibility to their advice to patients. NHS England's chief executive, Simon Stevens, has told NHS staff to join gyms and weight loss clubs to fight against obesity. But as Margaret McCartney points out, fat doctors are patients too (doi:10.1136/ bmj.g6464). We shouldn't assume that they are not thinking about their weight and wanting to do something about it, she says. "Those of us who have gained, lost, gained, lost, and gained weight again are only too aware of our failings."

Working conditions don't help. Shift working is a risk factor for obesity, made worse by the invasion by fast food outlets selling energy dense food on hospital concourses. Rather than judging individual colleagues we should concentrate on developing healthier working environments, McCartney says. We should demand better quality food outlets in hospitals and push for reasonable work schedules, regular meal and break times, and initiatives that promote cycling or walking to work. This brings me to our third role as doctors in the fight against obesity: understanding and acting on the causes. This is complicated by controversy and uncertainty. What is a healthy diet? Is a calorie just a calorie or does the source of the energy and the type and quality of the food matter, as a recent commentary in Public Health Nutrition suggests (doi:10.1017/ S1368980014002559). And what is the right way to promote healthy diets? Most people (except the food industry and its political allies) are now saying that this cannot be left to economic interests alone. A report from the US consultancy firm McKinsey, published this week, concludes that voluntary responsibility deals are not working and that regulation may be needed (http://bit.ly/1piYh8A). And the World Obesity Federation and UK Health Forum have joined forces to call on the World Health Organization to establish a global framework treaty similar to the treaty on tobacco control, to reduce saturated fats, added sugar, and salt in food and to control the marketing of highly processed foods (doi:10.1136/bmj.g6851). Doctors need to be in on this debate. It looks like nutrition is more important than some of us thought.

Cite this as: BMJ 2014;349:97255

(๑) BMJ Publishing Group Ltd 2014 